Few-Body Systems manuscript No.

(will be inserted by the editor)

Bernhard U. Musch

\title{
Studying the Sivers and Boer-Mulders function with Lattice QCD
}

Received: date / Accepted: date

\begin{abstract}
Transverse momentum dependent parton distribution functions (TMDs) characterize the intrinsic momentum distribution of quarks inside the nucleon. However, they also encode final or initial state interactions of the processes in which they are measured, such as semi-inclusive deep inelastic scattering (SIDIS) or the Drell-Yan process (DY). Consequently certain TMDs are process-dependent and predicted to be equal but opposite in sign for SIDIS and DY. Extending our method on the lattice to non-local operators with U-shaped Wilson lines, we can study these naively time-reversal odd TMDs, in particular the Sivers- and the Boer-Mulders function. We express our results in terms of Fourier-transformed TMDs that appear naturally in the Fourier transformed cross section of, e.g., SIDIS, and in Bessel-weighted asymmetries. We discuss the method, its limitations and preliminary results from an exploratory calculation using lattices generated by the MILC and LHP collaborations.
\end{abstract}

Keywords transverse momentum $\cdot$ lattice $\cdot$ QCD $\cdot$ Sivers $\cdot$ Boer-Mulders

\section{Introduction}

Processes such as Drell-Yan (DY) or in semi-inclusive deep inelastic scattering (SIDIS) can be used to study the intrinsic motion of quarks inside protons or neutrons, described by non-perturbative objects called transverse momentum dependent parton distribution functions (TMDs), see chapter 2 of [8] for a review. In SIDIS it is the measurement of a momentum $P_{h}$ of one of the produced hadrons that allows us to extract this information, see Fig. 1 and, e.g., Ref. [5] for a review of SIDIS and its kinematics. Due to

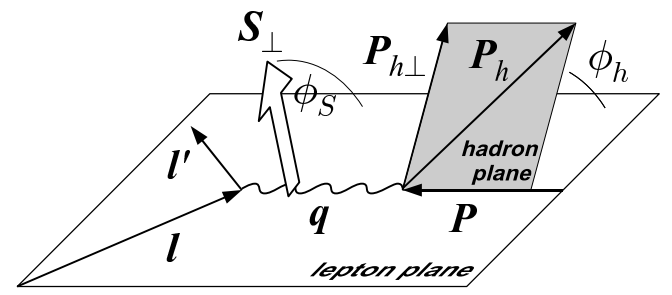

Fig. 1 Kinematics of the SIDIS process. The in- and out-going lepton momenta are $l$ and $l^{\prime}$, respectively. The momentum transfer is $q$. The target nucleon carries momentum $P$ and its transverse spin components are labelled $\boldsymbol{S}_{\perp}$. The momentum of the measured hadron $P_{h}$ has transverse components $\boldsymbol{P}_{h \perp}$, which define an angle $\phi_{h}$ with the lepton plane.

B. Musch

Jefferson Lab

Tel.: +757-269-2869

Fax: +757-269-7002

E-mail: bmusch@jlab.org 
initial state interactions (in DY) or final state interactions (in SIDIS) [10] whose theoretical explanation is deeply connected to the principle of gauge invariance, the operator definition of TMD PDFs is to a certain extent process-dependent. This leads to the prediction that so-called naively time-reversal odd (T-odd) TMD PDFs differ in sign for SIDIS and DY [12]. The $T$-odd distributions at leading twist are thought to be responsible for large single-spin asymmetries observed in experiment, see, e.g., [1]. Here we address them using lattice QCD. In previous lattice studies of TMD PDFs [20; 26], a simplified, "process-independent" operator geometry was chosen that does not strictly correspond to the definition of TMD PDFs appearing in the description of SIDIS or DY, and that does not feature T-odd TMD PDFs. Here we go beyond this simplification and show preliminary results obtained with a "processdependent" operator geometry that may ultimately allow quantitative comparisons to experimental SIDIS or DY results. Before discussing the method and preliminary results of our calculations, we show that the distributions in Fourier space obtained on the lattice could also be accessed fairly directly in phenomenology and appear naturally, e.g., in the context of Bessel-weighted asymmetries [7].

\section{The SIDIS cross section and Bessel-weighted asymmetries}

To convert the convolutions of TMD PDFs and TMD FFs in the SIDIS cross section into products, one can perform a multipole expansion and a subsequent Fourier transform of the cross section with respect to the transverse components $\boldsymbol{P}_{h \perp}$ of the hadron momentum. In general, we can write a transverse momentum dependent coss section $\sigma\left(\left|\boldsymbol{P}_{h \perp}\right|, \phi_{h}\right)$ in the form

$$
\begin{aligned}
\sigma\left(\left|\boldsymbol{P}_{h \perp}\right|, \phi_{h}\right) & =\int \frac{d^{2} \boldsymbol{b}_{T}}{(2 \pi)^{2}} e^{-i \boldsymbol{P}_{h \perp} \cdot \boldsymbol{b}_{T}} \tilde{\sigma}\left(\boldsymbol{b}_{T}\right) \\
& =\int \frac{d\left|\boldsymbol{b}_{T}\right|}{2 \pi}\left|\boldsymbol{b}_{T}\right| \int_{0}^{2 \pi} \frac{d \phi_{b}}{2 \pi} e^{-i\left|\boldsymbol{P}_{h \perp}\right|\left|\boldsymbol{b}_{T}\right| \cos \left(\phi_{h}-\phi_{b}\right)} \sum_{n=-\infty}^{\infty} e^{i n \phi_{b}} \tilde{\sigma}_{n}\left(\left|\boldsymbol{b}_{T}\right|\right) \\
& =\sum_{n=-\infty}^{\infty} e^{i n \phi_{h}} \int \frac{d\left|\boldsymbol{b}_{T}\right|}{2 \pi}\left|\boldsymbol{b}_{T}\right|(-i)^{n} J_{n}\left(\left|\boldsymbol{P}_{h \perp}\right|\left|\boldsymbol{b}_{T}\right|\right) \tilde{\sigma}_{n}\left(\left|\boldsymbol{b}_{T}\right|\right) .
\end{aligned}
$$

where $\tilde{\sigma}\left(\boldsymbol{b}_{T}\right)=\sum_{n=-\infty}^{\infty} e^{i n \phi_{b}} \tilde{\sigma}_{n}\left(\left|\boldsymbol{b}_{T}\right|\right)$ is a two-dimensional multipole expansion of the cross section in Fourier space. The $n^{\text {th }}$ harmonic in $\phi_{h}$ is accompanied by the $n^{\text {th }}$ Bessel function of the first kind $J_{n}$. Rewriting the cross section of SIDIS (see, e.g., Ref. [5]) in this form, we get (similar as in the work of Ref. [21] but in polar coordinates)

$$
\begin{aligned}
& \frac{d \sigma}{d x d y d \phi_{S} d z d \phi_{h}\left|\boldsymbol{P}_{h \perp}\right| d\left|\boldsymbol{P}_{h \perp}\right|} \\
& \approx \frac{\alpha^{2}}{y Q^{2}}\left(1+(1-y)^{2}\right) \sum_{a} e_{a}^{2} \int \frac{d\left|\boldsymbol{b}_{T}\right|}{(2 \pi)}\left|\boldsymbol{b}_{T}\right| \mathcal{S}_{\sigma}\left(\boldsymbol{b}_{T}^{2}\right)\{ \\
& J_{0}\left(\left|\boldsymbol{b}_{T}\right|\left|\boldsymbol{P}_{h \perp}\right|\right) H_{U U, T}\left(Q^{2}\right) \tilde{f}_{1, a}^{(0)}\left(x, z^{2} \boldsymbol{b}_{T}^{2}\right) \tilde{D}_{1, a}^{(0)}\left(z, \boldsymbol{b}_{T}^{2}\right) \\
&-\left|\boldsymbol{S}_{\perp}\right| \sin \left(\phi_{h}-\phi_{S}\right) J_{1}\left(\left|\boldsymbol{b}_{T}\right|\left|\boldsymbol{P}_{h \perp}\right|\right) H_{U T, T}^{\sin \left(\phi_{h}-\phi_{S}\right)}\left(Q^{2}\right) z M\left|\boldsymbol{b}_{T}\right| \tilde{f}_{1 T, a}^{\perp(1)}\left(x, z^{2} \boldsymbol{b}_{T}^{2}\right) \tilde{D}_{1, a}^{(0)}\left(z, \boldsymbol{b}_{T}^{2}\right) \\
&+\langle 16 \text { more terms }\rangle,
\end{aligned}
$$

where we have used the kinematic variables $Q^{2} \equiv-q^{2}, M^{2}=P^{2}, x \approx x_{B} \equiv Q^{2} / P \cdot q, y=P \cdot q / P \cdot l$, and $z \approx z_{h} \equiv P \cdot P_{h} / P \cdot q$ and assume $M \ll Q,\left|\boldsymbol{P}_{h \perp}\right| \ll z Q$. The sum $\sum_{a}$ runs over quark flavors $a$ and $e_{a}$ is the corresponding electric charge of the quark. Note that there is only a finite number of multipoles in the SIDIS cross section; the Bessel function of highest order is $J_{3}$. Here we show only two terms in the cross section and omit for now regularization parameters needed beyond tree level, see Ref. [7] and references therein for more details. The functions $\tilde{f}_{1, a}^{(0)}$ and $\tilde{f}_{1 T, a}^{\perp(1)}$ are examples of Fourier transformed TMD PDFs, while $\tilde{D}_{1, a}^{(0)}$ is a Fourier transformed fragmentation function. For a TMD PDF 
generically called $f$ and a TMD FF generically called $D$ the quantities in Fourier space are defined as

$$
\begin{array}{r}
\tilde{f}^{(n)}\left(x, \boldsymbol{b}_{T}^{2}\right)=\frac{2 \pi n !}{\left(M^{2}\right)^{n}} \int d\left|\boldsymbol{p}_{T}\right|\left|\boldsymbol{p}_{T}\right|\left(\frac{\left|\boldsymbol{p}_{T}\right|}{\left|\boldsymbol{b}_{T}\right|}\right)^{n} J_{n}\left(\left|\boldsymbol{b}_{T}\right|\left|\boldsymbol{p}_{T}\right|\right) f\left(x, \boldsymbol{p}_{T}^{2}\right), \\
\tilde{D}^{(n)}\left(z, \boldsymbol{b}_{T}^{2}\right)=\frac{2 \pi n !}{\left(z^{2} M_{h}^{2}\right)^{n}} \int d\left|\boldsymbol{K}_{T}\right|\left|\boldsymbol{K}_{T}\right|\left(\frac{\left|\boldsymbol{K}_{T}\right|}{\left|\boldsymbol{b}_{T}\right|}\right)^{n} J_{n}\left(\left|\boldsymbol{b}_{T}\right|\left|\boldsymbol{K}_{T}\right|\right) D\left(z, \boldsymbol{K}_{T}^{2}\right) .
\end{array}
$$

The soft factor $\mathcal{S}_{\sigma}\left(\boldsymbol{b}_{T}^{2}\right)$ accounts for the collective effect of soft momentum gluons not associated with either the distribution or fragmentation part of the process $[15 ; 13 ; 16 ; 22 ; 23 ; 3]$. Depending on the factorization framework, it appears explicitly in the cross section as in, e.g., Refs. $[22 ; 23]$ and as shown in Eq. (2), or it is completely absorbed in the definition of TMD PDFs and TMD FFs, see Refs. [13; 3]. At tree level, the soft factor and the hard parts $H_{\dddot{X} Y, Z}\left(Q^{2}\right)$ are unity.

An application of this formalism that could be phenomenologically useful are Bessel-weighted asymmetries. Projecting on suitable Fourier-Bessel modes and spin-polarizations, we can access individual terms in the cross section Eq. (2). In ratios of such terms the soft factor $\mathcal{S}_{\sigma}\left(\boldsymbol{b}_{T}\right)$ cancels, along with other multiplicative renormalization factors. In general, Bessel-weighted asymmetries are of the form

$$
A^{W_{1}} \equiv 2 \frac{\int d\left|\boldsymbol{P}_{h \perp}\right| d \phi_{h} d \phi_{S} W_{1}\left(\phi_{h},\left|\boldsymbol{P}_{h \perp}\right|\right) d \sigma}{\int d\left|\boldsymbol{P}_{h \perp}\right| d \phi_{h} d \phi_{S} W_{0}\left(\phi_{h},\left|\boldsymbol{P}_{h \perp}\right|\right) d \sigma}
$$

where the weight $W_{0}$ in the denominator is in general chosen to be $W_{0}\left(\phi_{h},\left|\boldsymbol{P}_{h \perp}\right|\right)=J_{0}\left(\left|\boldsymbol{P}_{h \perp}\right|\left|\boldsymbol{b}_{T}\right|\right)$ in order to project onto the unpolarized monopole contribution. As an example, the Bessel-weighted Sivers asymmetry is given by

$$
A_{U T}^{2 \sin \left(\phi_{h}-\phi_{s}\right) J_{1}\left(\left|\boldsymbol{P}_{h \perp} \| \boldsymbol{b}_{T}\right|\right) /\left(z M\left|\boldsymbol{b}_{T}\right|\right)}=-2 \frac{\sum_{a} e_{a}^{2} H_{U T, T}^{\sin \left(\phi_{h}-\phi_{S}\right)}\left(Q^{2}\right) \tilde{f}_{1 T, a}^{\perp(1)}\left(x, z^{2} \boldsymbol{b}_{T}^{2}\right) \tilde{D}_{1, a}^{(0)}\left(z, \boldsymbol{b}_{T}^{2}\right)}{\sum_{a} e_{a}^{2} H_{U U, T}\left(Q^{2}\right) \tilde{f}_{1, a}^{(0)}\left(x, z^{2} \boldsymbol{b}_{T}^{2}\right) \tilde{D}_{1, a}^{(0)}\left(z, \boldsymbol{b}_{T}^{2}\right)},
$$

Note that $\left|\boldsymbol{b}_{T}\right|$ enters the weights $W_{0}$ and $W_{1}$ as a free parameter that we can scan over a whole range in order to compare the transverse momentum dependence of the distributions in the numerator and denominator relative to each other (in Fourier space). As we will see later, on the operator level, $\left|\boldsymbol{b}_{T}\right|$ controls the space-like distance between quark fields in the correlation functions we measure. The Bessel-weighted asymmetries are a natural extension of conventional weighted asymmetries [24;6] with weights $W_{1}$ proportional to powers of $\left|\boldsymbol{P}_{h \perp}\right|$. For the above example, the weights become in this limit $W_{0}=1, W_{1}=\sin \left(\phi_{h}-\phi_{s}\right)\left|\boldsymbol{P}_{h \perp}\right| /(z M)$, and the Fourier transformed TMD PDFs and TMD FFs at $\left|\boldsymbol{b}_{T}\right|=0$ coincide with conventional transverse momentum moments:

$$
\tilde{f}^{(n)}(x, 0)=f^{(n)}(x) \equiv \int d^{2} \boldsymbol{p}_{T}\left(\frac{\boldsymbol{p}_{T}^{2}}{2 M^{2}}\right)^{n} f\left(x, \boldsymbol{p}_{T}\right) .
$$

An analogous equation holds for the fragmentation functions $D$.

One reason why it can be advantageous to analyze Bessel-weighted asymmetries at non-zero $\left|\boldsymbol{b}_{T}\right|$ rather than conventional asymmetries is the observation that in very important cases the defining integrals of transverse momentum moments $f^{(n)}(x)$ in the above equation are divergent due to the perturbatively predictable contribution from the tail of the TMD PDFs at large $\left|\boldsymbol{p}_{T}\right|$ [4]. The use of a non-zero $\left|\boldsymbol{b}_{T}\right|$ can be understood as a natural way of regularizing these divergences. A second, related advantage is that the Fourier-Bessel transform of the cross section at non-zero $\left|\boldsymbol{b}_{T}\right|$ puts more emphasis on the low- $\left|\boldsymbol{P}_{h \perp}\right|$ contributions than the traditional weights. Moreover, as we will see in the following, the quantities in Fourier space are more suitable to compare with results from lattice QCD.

\section{TMD PDFs on the operator level}

The correlator that defines TMD PDFs for SIDIS and DY can be written in general as

$$
\Phi^{[\Gamma]} \equiv \frac{1}{2} \int \frac{d^{4} b}{(2 \pi)^{4}} e^{i p \cdot b} \overbrace{\frac{\langle P, S|\bar{q}(0) \Gamma \mathcal{U}[0, \eta v, \eta v+b, b] q(b)| P, S\rangle}{\widetilde{\mathcal{S}}_{\Phi}\left(b^{2}, \ldots\right)}}^{\equiv \widetilde{\Phi}_{\text {unsubtr. }}^{[\Gamma]}(b, P, S, \eta v, \mu)}
$$




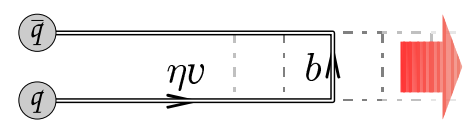

Fig. 2 Geometry of the staple shaped gauge link. On the lattice, we compute the SIDIS/DY limits $\eta \rightarrow \pm \infty$ by increasing the extent of the staple step by step, as indicated by the dashed lines and the arrow.

where $P$ and $S$ are momentum and spin of the nucleon, $p$ is the quark momentum, $\Gamma$ is a Dirac matrix and $\mu$ is a renormalization scale. Note that $b$ corresponds to the variable $l$ in the notation of Refs. $[20 ; 26]$. The precise definition of the soft factor $\widetilde{\mathcal{S}}_{\Phi}$ varies in different theory frameworks, see, e.g., $[23 ; 11 ; 3]$. However, it cancels explicitly in the quantities for which we show results.

We use light cone coordinates and consider a fast nucleon, $P_{\perp}=0, P^{+} \gg M$. Integrating over the suppressed component $p^{-}$of the intrinsic quark momentum, the decomposition of the correlator $[25 ; 19]$ yields

$$
\int d p^{-} \Phi^{\left[\gamma^{+}\right]}=f_{1}\left(x, \boldsymbol{p}_{T}^{2} ; \hat{\zeta}, \eta, \ldots\right)-\frac{\boldsymbol{\epsilon}_{i j} \boldsymbol{p}_{i} \boldsymbol{S}_{j}}{m_{N}} f_{1 T}^{\perp}\left(x, \boldsymbol{p}_{T}^{2} ; \hat{\zeta}, \eta, \ldots\right)
$$

for a projection $\Gamma=\gamma^{+}$on leading twist and for transverse nucleon polarization $\boldsymbol{S}_{T}$. Here $f_{1}$ and the Sivers function $f_{1 T}^{\perp}$ [27] are the two TMD PDFs that describe the corresponding distribution of quarks with respect to the longitudinal momentum fraction $x \equiv p^{+} / P^{+}$and the transverse momentum $\boldsymbol{p}_{T}$ of the quark. The Wilson line $\mathcal{U}$ in Eq. (7) ensures gauge invariance and effectively represents gluon exchanges in initial or final state interactions. As illustrated in Fig. 2, it is composed of two parallel straight sections along the direction $v \approx \hat{n}_{-}$and a gauge link bridging the (transverse) gap at the far ends. For SIDIS, the extent $\eta$ of the staple is $+\infty$, while for DY the staple extends in the opposite direction, $\eta=-\infty$. The T-odd Sivers function $f_{1 T}^{\perp}$ differs for SIDIS and DY, $f_{1 T}^{\perp}(\eta=+$ $\infty)=-f_{1 T}^{\perp}(\eta=-\infty)$, while $f_{1}(\eta=+\infty)=f_{1}(\eta=-\infty)$ exhibits $T$-even behavior. Another leadingtwist T-odd TMD PDF is the Boer-Mulders function $h_{1}^{\perp}[6]$, which describes correlations in $\boldsymbol{p} \times \boldsymbol{s}$ of quarks polarized transversely along $\boldsymbol{s}_{T}$ in an unpolarized nucleon. For brevity, we focus on the Sivers function in this article. Employing a direction $v$ off the light cone $\hat{n}_{-}$direction is one way to regularize rapidity divergences in the correlator, see, e.g., Refs. [17; 23]. Taking $v$ space-like [16; 3; 14] also opens up the possibility to perform lattice calculations. The TMD PDFs in this framework depend on additional parameters $\hat{\zeta}, \eta, \ldots$ not shown in the previous section. Important for our work is the parameter describing the direction $v$ of the gauge link, here introduced as a dimensionless quantity $\hat{\zeta} \equiv v \cdot P / \sqrt{\left|v^{2}\right| P^{2}}$. At large enough values of $\hat{\zeta}$, the $\hat{\zeta}$-dependence of TMD PDFs can be obtained from evolution equations, see, e.g., Refs. [21; 3]. Dependencies of the TMD PDFs on further regularization or renormalization scales have been indicated by the dots and cancel in the quantities we consider.

\section{Lattice calculations}

In previous lattice studies of TMD PDFs [20;26], a direct, straight gauge link $\mathcal{U}[0, b]$ was employed, corresponding to $\eta=0$ in Eq. (7). We make use of the same lattice at $m_{\pi} \approx 500 \mathrm{MeV}$ [2] and the same techniques as in these earlier works, except that we now implement the staple-shaped operator geometry of Fig. 2. We also improve our statistics using the new arrangement of nucleon sources and coherent sinks of Ref. [9]. In essence, we calculate $\widetilde{\Phi}_{\text {unsubtr. }}^{[\Gamma]}(b, P, S, \eta v, \mu)$ directly for a large selection of lattice vectors $\boldsymbol{b}, \boldsymbol{P}$ and $\eta \boldsymbol{v}$. As before, we restrict the operator to have no extent in Euclidean time direction. Consequently, $b$ and $v$ can only have spatial components on the lattice. For a given lattice nucleon threemomentum $\boldsymbol{P}^{\text {lat }}$, the regularization parameter $\hat{\zeta}$ is thus limited by $\hat{\zeta} \leq\left|\boldsymbol{P}^{\text {lat }}\right| / M^{2}$. The translation of the results obtained in the lattice frame to the TMD PDF language is established through a parametrization of $\widetilde{\Phi}_{\text {unsubtr. }}^{[\Gamma]}$ in terms of Lorentz-invariant amplitudes $\widetilde{A}_{i}$ and $\widetilde{B}_{i}$, analogously to Ref. [19] but in $b$-space. These amplitudes can then be converted to the TMD PDFs in Fourier space already encountered in section 2. As argued before, in the limit $\left|\boldsymbol{b}_{T}\right| \rightarrow 0$ we obtain moments of TMD PDFs, which sometimes have an intuitive interpretation. For example, the quantity $\left\langle\boldsymbol{p}_{y}\right\rangle_{T U}(x) \equiv M f_{1 T}^{\perp(1)}(x) / f_{1}^{(0)}(x)$, has an interpretation as the average transverse momentum in transverse $y$-direction carried by the quarks inside a nucleon polarized in transverse $x$-direction. Similar quantities, generalized to non-zero $\left|\boldsymbol{b}_{T}\right|$, are accessible on the lattice. Here we restrict ourselves to $x$-integrated TMD PDFs $\tilde{f}^{[1](n)}\left(\boldsymbol{b}_{T}^{2}\right) \equiv$ 
a)

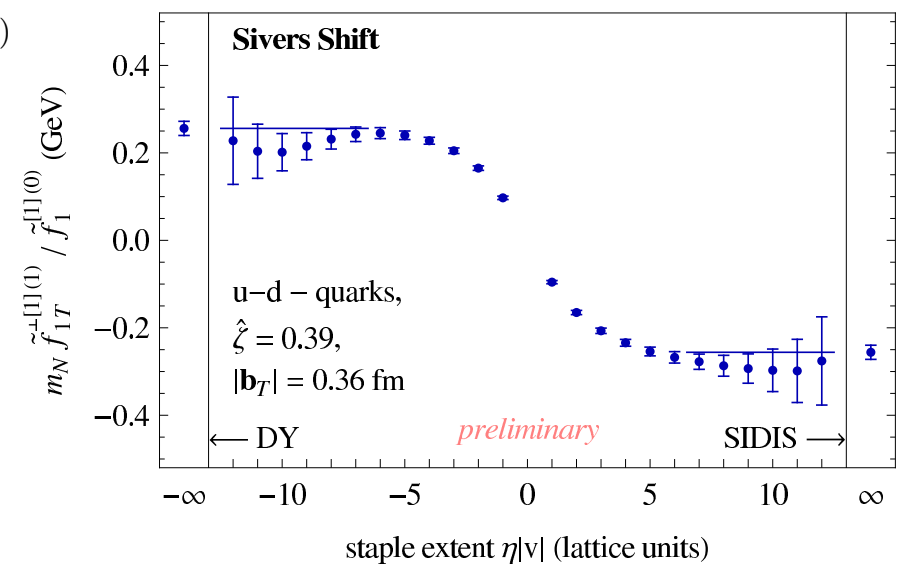

b)

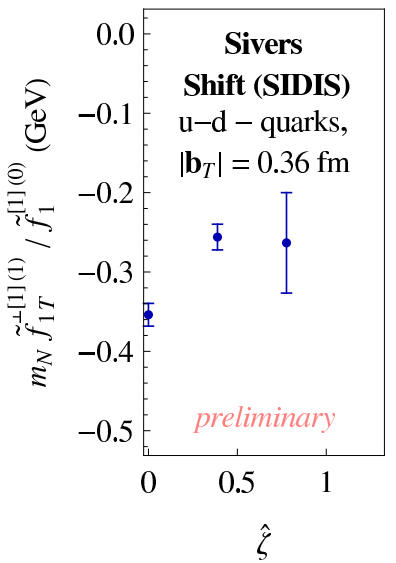

Fig. 3 Generalized Sivers shift of up-down quarks (isovector) on the $20^{3} \times 64$ lattice at a pion mass $m_{\pi} \approx$ $500 \mathrm{MeV}$ and a lattice spacing of $a \approx 0.12 \mathrm{fm}$. a) results as a function of the staple extent $\eta$. A simple estimate of the SIDIS/DY values at $\eta \rightarrow \pm \infty$ is obtained from a fit of an odd but otherwise constant function to the data at $|\eta v| \geq 7 a$. Potentially significant systematic uncertainties in this procedure have not been taken into account in this preliminary analysis. b) Extracted SIDIS results for several values of $\hat{\zeta}$.

$\int_{-1}^{1} d x \tilde{f}^{[1](n)}\left(x, \boldsymbol{b}_{T}^{2}\right)$, which can be obtained from the amplitudes at $b \cdot P=0$ :

$$
\left.\frac{1}{2} \widetilde{\Phi}_{\text {unsubtr. }}^{\left[\gamma^{+}\right]}\right|_{\substack{b \cdot P=0 \\ S=0}}=P^{+} \underbrace{\left(\widetilde{A}_{2}+R(\hat{\zeta}) \widetilde{B}_{1}\right)}_{\widetilde{\mathcal{S}}_{\Phi}\left(b^{2}, \ldots\right) \tilde{f}_{1}^{[1](0)}\left(\boldsymbol{b}_{T}^{2}\right)}-i M P^{+} \boldsymbol{\epsilon}_{i j} \boldsymbol{b}_{i} \boldsymbol{S}_{j} \underbrace{\left(\widetilde{A}_{12}-R(\hat{\zeta}) \widetilde{B}_{8}\right)}_{\widetilde{\mathcal{S}}_{\Phi}\left(b^{2}, \ldots\right) \tilde{f}_{1 T}^{\perp[1](1)}\left(\boldsymbol{b}_{T}^{2}\right)},
$$

where $R(\hat{\zeta}) \equiv 1-\left(1+\hat{\zeta}^{-2}\right)^{1 / 2}$. We thus can construct a ratio which looks similar to the average momentum $\left\langle\boldsymbol{p}_{y}\right\rangle_{T U}(x)$, but is formed from $x$-integrated distributions and generalized to non-zero $\boldsymbol{b}_{T}$, in the following called the (generalized) Sivers shift:

$$
\left\langle\boldsymbol{p}_{y}\right\rangle_{T U}\left(\left|\boldsymbol{b}_{T}\right|\right) \equiv M \frac{\tilde{f}_{1 T}^{\perp[1](1)}\left(\boldsymbol{b}_{T}^{2}\right)}{\tilde{f}_{1}^{[1](0)}\left(\boldsymbol{b}_{T}^{2}\right)}=-\left.M \frac{\widetilde{A}_{12}-R(\hat{\zeta}) \widetilde{B}_{8}}{\widetilde{A}_{2}+R(\hat{\zeta}) \widetilde{B}_{1}}\right|_{b \cdot P=0}
$$

Analogously, the "Boer-Mulders shift" can be constructed using $\tilde{h}_{1}^{\perp[1](1)}$ instead of $\tilde{f}_{1 T}^{\perp[1](1)}$. The soft factor and multiplicative renormalization factors cancel in the above ratio. However, the dependence on the rapidity cutoff parameter $\hat{\zeta}$ (not shown in the arguments) survives. Figure 3 a) demonstrates how the SIDIS or DY Sivers shift can be read off from the plateau reached at large positive or negative $\eta$, respectively. The extraction of these asymptotic values is still preliminary and lacks an estimate of systematic errors. In Fig. $3 \mathrm{~b}$ ), we plot the extracted SIDIS results as a function of $\hat{\zeta}$ and find indications of a strong $\hat{\zeta}$-dependence at the rather low values of $\hat{\zeta}$ presently accessible to us. A major future challenge is to generate statistically well-determined results at higher values of $\hat{\zeta}$ and to make contact with the $\hat{\zeta}$-evolution predicted by perturbative QCD.

\section{Conclusion}

We have shown preliminary results of a lattice study for the ratio $\tilde{f}_{1 T}^{\perp[1](1)} / \tilde{f}_{1}^{[1](0)}$ of the Sivers function to the unpolarized distribution in Fourier-space, albeit currently only available for rather small values of the Collins-Soper evolution parameter $\hat{\zeta}$ and at a pion mass $m_{\pi} \approx 500 \mathrm{MeV}$. The lattice results are obtained in Fourier space. Bessel-weighted asymmetries and related quantities might ultimately provide an interface to compare these results rather directly to phenomenology.

Acknowledgements Thanks are due to Daniel Boer, Leonard Gamberg, Philipp Hägler, John Negele, Andreas Schäfer and Alexei Prokudin for feedback and support, and to Elke Aschenauer, Harut Avakian, Mert 
Aybat, Vladimir Braun, Matthias Burkardt, John Collins, Markus Diehl, Aram Kotzinian, Andreas Metz, Piet Mulders, Dru Renner, Ted Rogers and Feng Yuan for helpful discussions. We are grateful to the LHP and MILC collaborations, for providing gauge configurations and propagators used in this work [2; 9]. Our software uses the Chroma-library [18], and we use USQCD computing resources at Jefferson Lab. Authored by Jefferson Science Associates, LLC under U.S. DOE Contract No. DE-AC05-06OR23177. The U.S. Government retains a non-exclusive, paid-up, irrevocable, world-wide license to publish or reproduce this manuscript for U.S. Government purposes.

\section{References}

1. Anselmino M, et al (2009) Sivers Effect for Pion and Kaon Production in Semi- Inclusive Deep Inelastic Scattering. Eur Phys J A39:89-100, DOI 10.1140/epja/i2008-10697-y, 0805.2677

2. Aubin C, et al (2004) Light hadrons with improved staggered quarks: approaching the continuum limit. Phys Rev D70:094,505, DOI 10.1103/PhysRevD.70.094505, hep-lat/0402030

3. Aybat SM, Rogers TC (2011) TMD Parton Distribution and Fragmentation Functions with QCD Evolution. Phys Rev D83:114,042, DOI 10.1103/PhysRevD.83.114042, 1101.5057

4. Bacchetta A, Boer D, Diehl M, Mulders PJ (2008) Matches and mismatches in the descriptions of semi- inclusive processes at low and high transverse momentum. JHEP 08:023, DOI 10.1088/11266708/2008/08/023, 0803.0227

5. Bacchetta A, et al (2007) Semi-inclusive deep inelastic scattering at small transverse momentum. JHEP 02:093, hep-ph/0611265

6. Boer D, Mulders PJ (1998) Time-reversal odd distribution functions in leptoproduction. Phys Rev D57:5780-5786, DOI 10.1103/PhysRevD.57.5780, hep-ph/9711485

7. Boer D, Gamberg L, Musch B, Prokudin A (2011) Bessel-Weighted Asymmetries in Semi Inclusive Deep Inelastic Scattering 1107.5294

8. Boer D, et al (2011) Gluons and the quark sea at high energies: distributions, polarization, tomography 1108.1713

9. Bratt JD, et al (2010) Nucleon structure from mixed action calculations using $2+1$ flavors of asqtad sea and domain wall valence fermions. Phys Rev D82:094,502, DOI 10.1103/PhysRevD.82.094502, 1001.3620

10. Brodsky SJ, Hwang DS, Schmidt I (2002) Final-state interactions and single-spin asymmetries in semiinclusive deep inelastic scattering. Phys Lett B530:99-107, DOI 10.1016/S0370-2693(02)01320-5, hep$\mathrm{ph} / 0201296$

11. Cherednikov IO, Stefanis NG (2008) Wilson lines and transverse-momentum dependent parton distribution functions: A renormalization-group analysis. Nucl Phys B802:146-179, DOI 10.1016/j.nuclphysb.2008.05.011, 0802.2821

12. Collins JC (2002) Leading-twist single-transverse-spin asymmetries: Drell- Yan and deep-inelastic scattering. Phys Lett B536:43-48, DOI 10.1016/S0370-2693(02)01819-1, hep-ph/0204004

13. Collins JC (2011) Foundations of Perturbative QCD. Cambridge University Press

14. Collins JC (2011) Foundations of Perturbative QCD. Cambridge University Press

15. Collins JC, Hautmann F (2000) Infrared divergences and non-lightlike eikonal lines in Sudakov processes. Phys Lett B472:129-134, DOI 10.1016/S0370-2693(99)01384-2, hep-ph/9908467

16. Collins JC, Metz A (2004) Universality of soft and collinear factors in hard- scattering factorization. Phys Rev Lett 93:252,001, DOI 10.1103/PhysRevLett.93.252001, hep-ph/0408249

17. Collins JC, Soper DE (1981) Back-to-back jets in QCD. Nucl Phys B193:381, DOI 10.1016/05503213(81)90339-4

18. Edwards RG, Joo B (2005) The Chroma software system for lattice QCD. Nucl Phys Proc Suppl 140:832, DOI 10.1016/j.nuclphysbps.2004.11.254, hep-lat/0409003

19. Goeke K, Metz A, Schlegel M (2005) Parameterization of the quark-quark correlator of a spin- $1 / 2$ hadron. Phys Lett B618:90-96, DOI 10.1016/j.physletb.2005.05.037, hep-ph/0504130

20. Hägler P, Musch B, Negele J, Schäfer A (2009) Intrinsic quark transverse momentum in the nucleon from lattice QCD. Europhys Lett 88:61,001, DOI 10.1209/0295-5075/88/61001, 0908.1283

21. Idilbi A, Ji Xd, Ma JP, Yuan F (2004) Collins-Soper equation for the energy evolution of transverse-momentum and spin dependent parton distributions. Phys Rev D70:074,021, DOI 10.1103/PhysRevD.70.074021, hep-ph/0406302

22. Ji Xd, Ma JP, Yuan F (2004) QCD factorization for spin-dependent cross sections in DIS and Drell-Yan processes at low transverse momentum. Phys Lett B597:299-308, DOI 10.1016/j.physletb.2004.07.026, hep-ph/0405085

23. Ji Xd, Ma Jp, Yuan F (2005) QCD factorization for semi-inclusive deep-inelastic scattering at low transverse momentum. Phys Rev D71:034,005, DOI 10.1103/PhysRevD.71.034005, hep-ph/0404183

24. Kotzinian AM, Mulders PJ (1997) Probing transverse quark polarization via azimuthal asymmetries in leptoproduction. Phys Lett B406:373-380, DOI 10.1016/S0370-2693(97)00708-9, hep-ph/9701330

25. Mulders PJ, Tangerman RD (1996) The complete tree-level result up to order 1/Q for polarized deepinelastic leptoproduction. Nucl Phys B461:197-237, DOI 10.1016/0550-3213(95)00632-X, hep-ph/9510301

26. Musch BU, Hägler P, Negele JW, Schäfer A (2011) Exploring quark transverse momentum distributions with lattice QCD. Phys Rev D83:094,507, DOI 10.1103/PhysRevD.83.094507, 1011.1213

27. Sivers DW (1990) Single spin production asymmetries from the hard scattering of point-like constituents. Phys Rev D41:83, DOI 10.1103/PhysRevD.41.83 